\title{
Stimulus Phase Locking of Cortical Oscillations for Rhythmic Tone Sequences in Rats
}

\author{
Takahiro Noda ${ }^{1,2 \dagger}$, Tomoki Amemiya ${ }^{3 \dagger}$, Tomoyo I. Shiramatsu ${ }^{1}$ and Hirokazu Takahashi ${ }^{1,3 *}$ \\ ${ }^{1}$ Research Center for Advanced Science and Technology, University of Tokyo, Tokyo, Japan, ${ }^{2}$ Institute of Neuroscience, \\ Technical University Munich, Munich, Germany, ${ }^{3}$ Graduate School of Information Science and Technology, University of \\ Tokyo, Tokyo, Japan
}

Humans can rapidly detect regular patterns (i.e., within few cycles) without any special attention to the acoustic environment. This suggests that human sensory systems are equipped with a powerful mechanism for automatically predicting forthcoming stimuli to detect regularity. It has recently been hypothesized that the neural basis of sensory predictions exists for not only what happens (predictive coding) but also when a particular stimulus occurs (predictive timing). Here, we hypothesize that the phases of neural oscillations are critical in predictive timing, and these oscillations are modulated in a band-specific manner when acoustic patterns become predictable, i.e., regular. A high-density microelectrode array $\left(10 \times 10\right.$ within $\left.4 \times 4 \mathrm{~mm}^{2}\right)$ was used to characterize spatial patterns of band-specific oscillations when a random-tone sequence was switched to a regular-tone sequence. Increasing the regularity of the tone sequence enhanced phase locking in a band-specific manner, notwithstanding the type of the

OPEN ACCESS

Edited by:

Isao Hasegawa,

Niigata University, Japan

Reviewed by:

Heather Read,

University of Connecticut, USA Drew Battenfield Headley,

Rutgers University, USA

*Correspondence:

Hirokazu Takahashi

takahashi@i.u-tokyo.ac.jp

${ }^{\dagger}$ These authors have contributed equally to this work.

Received: 30 March 2016 Accepted: 04 January 2017 Published: 26 January 2017

Citation:

Noda T, Amemiya T, Shiramatsu TI and Takahashi $H$ (2017) Stimulus Phase Locking of Cortical Oscillations for Rhythmic Tone Sequences in Rats.

Front. Neural Circuits 11:2. doi: 10.3389/fncir.2017.00002 regular sound pattern. Gamma-band phase locking increased immediately after the transition from random to regular sequences, while beta-band phase locking gradually evolved with time after the transition. The amplitude of the tone-evoked response, in contrast, increased with frequency separation with respect to the prior tone, suggesting that the evoked-response amplitude encodes sequence information on a local scale, i.e., the local order of tones. The phase locking modulation spread widely over the auditory cortex, while the amplitude modulation was confined around the activation foci. Thus, our data suggest that oscillatory phase plays a more important role than amplitude in the neuronal detection of tone sequence regularity, which is closely related to predictive timing. Furthermore, band-specific contributions may support recent theories that gamma oscillations encode bottom-up prediction errors, whereas beta oscillations are involved in top-down prediction.

Keywords: regular tone sequences, high-density microelectrode array, auditory cortex, oscillatory phase locking, rat

\section{INTRODUCTION}

Repeated, regular sound patterns that are acoustically distinct from noise are commonly observed in animal vocalization, human speech, and natural sound. Thus, detection of regular acoustic patterns is crucial for survival because such patterns probably have behavioral implications. Increasing evidence indicates that animals can inherently distinguish regular sound patterns. For 
instance, rodents can distinguish tone sequences with different tone orders (Toro and Trobalón, 2005; Murphy and Cook, 2008; Mondragón et al., 2009; Urcelay and Miller, 2010; Perry and Felsen, 2012; de la Mora and Toro, 2013), birds can not only learn rhythmic-arrhythmic discrimination but also distinguish different vocalization patterns with different syntactic rules (Hulse et al., 1984; Shinn-Cunningham et al., 2006; Abe and Watanabe, 2011; Schneider and Woolley, 2013), and primates can recognize and generalize tone patterns beyond specific pitches or stimulus lengths (Ravignani et al., 2013; Wilson et al., 2013, 2015).

Regularity detection in humans is so powerful that regular patterns can be very rapidly detected (i.e., within a few cycles) without any special attention to the acoustic environment (Chait et al., 2008; Jaunmahomed and Chait, 2012). The detection of regular acoustic patterns is accompanied by cortical responses, as measured non-invasively, e.g., transition from a random to a regular pattern is accompanied by an increase in magnetoencephalography (MEG) response (Chait et al., 2008). Neural activity upon the breaking of regular structure is characterized as mismatch negativity (Horváth et al., 2001). Furthermore, late electroencephalography (EEG) components are related to involuntary attention switches (Bendixen et al., 2007; Smith et al., 2010). In addition to these net changes in neural activity, local activity in primary and higher auditory cortical areas is probably involved in the detection of sound regularity and the integration of sequential auditory events (Griffiths et al., 1998; Mustovic et al., 2003). These observations suggest that the human sensory system is equipped with a powerful mechanism for the automatic prediction of forthcoming stimuli, which is used to detect regularity.

The neural mechanisms of sensory prediction have recently emerged a major topic of debate. Recent theories suggest that neural activity can be assessed to detect or predict either what sensory event would occur (predictive coding) or when a particular sensory event would occur (predictive timing) (Friston, 2005, 2009; Wacongne et al., 2011; Arnal and Giraud, 2012; Malmierca et al., 2015). Specifically, cortical gamma-band and beta-band oscillations are differently modulated during beat processing; this suggests that each band is differentially involved in predictive coding or predictive timing (Fujioka et al., 2009, 2015, 2012; Lee et al., 2013; Fries, 2015). Increasing evidence has led to the attractive hypothesis that gamma-band oscillations convey prediction errors regarding both predictive coding and timing in a bottom-up manner, whereas beta-band oscillations convey predictive timing in a top-down manner. For these oscillatory dynamics, entrainment in a specific band (i.e., phaselock to the structure of the stimulus stream) may be crucial for sensory selection involving predictive timing (Kruglikov and Schiff, 2003; Lakatos et al., 2007; Schroeder et al., 2008) or the build-up effect of auditory or visual objects (Busch et al., 2009; Busch and VanRullen, 2010; Schroeder et al., 2010; Giraud and Poeppel, 2012; Wimber et al., 2012; Noda et al., 2013b; Ossmy et al., 2015; Samaha et al., 2015; Vanvooren et al., 2015; Watrous et al., 2015). These band-specific phase entrainments are probably associated with neuronal detection and/or prediction of regular acoustic patterns. However, this possibility has not been investigated in detail.
We hypothesized that the phases of gamma-band and betaband oscillations are differentially modulated when sound patterns are predictable or regular, even under anesthesia. This may then serve as the neuronal signature of automatic detection and/or prediction of sound pattern regularity. We densely mapped the auditory cortex of rats under isofluraneinduced anesthesia and determined whether and how bandspecific oscillations entrain cortical population activity at the transition from random to regular patterns. We demonstrated that band-specific phase locking changes with sound pattern regularity irrespective of tone patterns, while the evoked response amplitude depends on tone patterns. This evolution of phase locking widely occurred over the auditory cortex irrespective of frequency bands, while modulation of evoked response amplitude was confined around the local foci of activation. These results support our hypothesis that neuronal oscillation in a particular band is critical for the representation of acoustic pattern regularity.

\section{MATERIALS AND METHODS}

This study was conducted in strict accordance with the "Guiding Principles for the Care and Use of Animals in the Field of Physiological Science" published by the Japanese Physiological Society. The experimental protocol was approved by the Committee on the Ethics of Animal Experiments at the Research Center for Advanced Science and Technology at the University of Tokyo (Permit Number: RAC130107). All surgeries were performed under isoflurane-induced anesthesia and animal suffering was minimized.

\section{Animal Preparation}

Seven adult male Wistar rats at postnatal week 7 were used in the experiments. Surgeries to expose the auditory cortex were carried out as reported previously (Takahashi et al., 2004, 2005; Shiramatsu et al., 2013). Animals were anesthetized using isoflurane (2.5-3.5\% at induction and $1.0-2.0 \%$ for maintenance) and held in a fixed position using a custom-made headholding device. Atropine sulfate $(0.1 \mathrm{mg} / \mathrm{kg})$ was administered at the beginning of the surgery and every $7 \mathrm{~h}$ to reduce the viscosity of bronchial secretions. A heating blanket was used to maintain body temperature at $\sim 37^{\circ} \mathrm{C}$. A skin incision was made at the beginning of the surgery under local anesthesia $(1 \%$ xylocaine, $0.3 \mathrm{~mL})$. The temporal muscle, cranium, and dura mater overlying the right auditory cortex were surgically removed, and the exposed cortical surface was filled with saline to prevent desiccation. Cerebral edema was minimized by drainage of cisternal cerebrospinal fluid. Respiratory rate, heart rate, and hind paw withdrawal reflexes were monitored to maintain a stable and adequate anesthetic level throughout the recording procedure. Additionally, a small craniotomy was performed near the bregma to embed a reference electrode with an electrical contact to the dura. The ground electrode was placed under the cervical neck skin. The right eardrum was ruptured and waxed to ensure unilateral sound inputs from the ear contralateral to the exposed cortex. 
All electrophysiological recordings were performed in a sound-attenuated room (AMC-4015; O'Hara and Co., Ltd., Tokyo, Japan). Microelectrode arrays with $10 \times 10$ recording site grids and inter-electrode distances of $400 \mu \mathrm{m}$ (ICS-96 Array; Blackrock Microsystems Inc., Salt Lake City, UT) were used as described previously (Noda et al., 2013b). Neural signals were simultaneously obtained from 96 electrodes (4 corner electrodes were excluded from the analysis). Electrode impedances were approximately $120 \mathrm{k} \Omega$ under $1-\mathrm{kHz}, 0.1-\mathrm{V}$ sinusoidal waves.

Neural signals were simultaneously amplified with a gain of 1000 and recorded using a neural data acquisition system (Cerebus data acquisition system; Cyberkinetics Inc., Salt Lake City, UT). Local field potentials (LFPs) and multiunit activities (MUAs) were obtained using digital bandpass filters of $0.3-500$ $\mathrm{Hz}$ and $0.25-7.5 \mathrm{kHz}$, and sampling frequencies of $1 \mathrm{kHz}$ and $30 \mathrm{kHz}$, respectively. The spatial distributions of click-evoked LFPs were first mapped on the cortical surface to identify the location of the auditory cortex. The largest focal activation in response to a click was regarded as the center of the anterior auditory cortex (Takahashi et al., 2005) and served as a landmark for the appropriate positioning of the electrode array. The arrays were inserted to a depth of approximately $700 \mu \mathrm{m}$ below the pial surface to measure LFPs.

\section{Stimulation}

Prior to the main experiments, a frequency response area (FRA) and a characteristic frequency (CF) were identified at each recording site using the MUA, as previously described (Takahashi et al., 2011; Funamizu et al., 2013). Recording sites with a CF were considered parts of the auditory cortex. Only these sites were included in the analyses of the following main experiments. Characterization of the CF is described in detail elsewhere. Briefly, we considered the number of spikes during the 5$45 \mathrm{~ms}$ post stimulus onset as a tone-evoked response. The CF at each site was then determined as the frequency at which the test tones evoked a response for the lowest intensity or as the frequency evoking the largest response at a $20-\mathrm{dB}$ SPL (sound pressure level in decibels re $20 \mu \mathrm{Pa}$ ), which was the minimum intensity used in this experiment. Multiunit spikes were detected as threshold-crossing events with negative thresholds ( -5.65 root mean square [RMS] from the average of the MUA recordings). Test stimuli were tone bursts of 30-ms duration with 5-ms linear rise/fall ramps. The test frequencies ranged between 1.6 and $64 \mathrm{kHz}$ with 1/3-octave increments. Test stimuli had intensities between 20 and $80 \mathrm{~dB}$ SPL with $10-\mathrm{dB}$ increments. Since there were 18 test frequencies and 7 intensities used, 126 test tones were used in total. Each tone was presented 20 times in a pseudorandom order with an inter-tone interval of $600 \mathrm{~ms}$.

Figure 1 shows the test stimuli used in the main experiments. Five tone sequences were prepared in total. Patterns A-D were the regular sequences in the main test and the random pattern was used as a control sequence. The inter-sequence interval consisted of $30 \mathrm{~s}$ of silence. Each sequence consisted of 8, 12.5, 20 , or $32-\mathrm{kHz}$ element tones with inter-tone intervals of $200 \mathrm{~ms}$. All of the tones appeared with equal probabilities (25\%). Each element tone was a tone burst of $70 \mathrm{~dB}$ SPL and 50-ms duration and had 5-ms linear rise/fall ramps. Each tone sequence started with an adaptation period of $16 \mathrm{~s}$ (80 tones), which was followed by a 16 -s test period ( 80 tones). During the adaptation period, each element tone appeared in a pseudo-random order. During the test period, a particular order of 4 element tones was repeated in 4 test sequences (patterns A-D). Each element tone continued to appear in a pseudo-random order in the control sequence (random pattern). To investigate the build-up effect of neural responses, the adaptation and test periods were divided into 3 sub-periods of $8 \mathrm{~s}$ with an overlap of $4 \mathrm{~s}$ (A1-A3 and T1-T3).

A speaker (EAS-10TH800; Panasonic Corp., Osaka, Japan) positioned $10 \mathrm{~cm}$ anterior to the left (contralateral) ear delivered the sounds. Prior to the experiments, all of the frequencies and intensities of the test stimuli were calibrated at the pinna using a microphone (4939; Brüel and Kjær, Nærum, Denmark) and a spectrum analyzer (CF-5210; Ono Sokki Co., Ltd., Kanagawa, Japan). A function generator (WF1974; NF Corp., Kanagawa, Japan) was used to generate test tones for FRA characterization. Tone sequences in the main experiments were generated using a 2-M sample/second 16-bit digital to analog converter (NI USB6361; National Instruments Corp., Austin, TX) and attenuated by $20 \mathrm{~dB}$ using an attenuator (PA5; Tucker \& Davis Technologies Inc., Alachua, FL).

\section{Data Analysis}

Tone-evoked LFP amplitudes and phase locking were investigated. All data were analyzed using custom-made Matlab codes (Mathworks Inc., Natick, MA).

For each element tone delivered during the test period for a given sequence, the peak amplitudes of evoked LFPs, termed P1 (Figure 2A), were quantified at each site and spatially mapped as functions of test frequency. The differences in the grand-averaged P1s during the test period for each test sequence (patterns AD) with respect to the control sequence (random pattern) were defined as $\Delta \mathrm{P} 1 \mathrm{~s}$ (Figure 2Bi). Positive $\Delta \mathrm{P} 1 \mathrm{~s}$ indicate that the $\mathrm{P} 1$ amplitude of a regular tone sequence is larger than that of a random sequence.

In addition to the P1 amplitudes, phase locking of bandspecific LFPs was quantified. The measured LFPs were split into four frequency bands by digital band-pass filtering. These bands are as follows: alpha, $8-14 \mathrm{~Hz}$; beta, $14-30 \mathrm{~Hz}$; low-gamma, 30-40 Hz; and high-gamma, 60-80 Hz. The digital filters used were linear-phase finite-impulse response (FIR) filters, which were designed using the Parks-McClellan algorithm (Matlab; Mathworks Inc., Natick, MA) such that the attenuations at $90 \%$ of the low band edge and $110 \%$ of the high band edge were 40 dB. The Matlab function "filtfilt" was used to apply a non-causal zero phase filter. Filtered signals were converted into analytic signals of an instantaneous phase using the Hilbert transform (Figure 2A). Phase locking was then quantified using inter-trial phase coherence (ITPC) on the basis of the phase distribution across trials:

$$
\operatorname{ITPC}(t)=\left|\frac{1}{K} \sum_{k=1}^{K} \exp \left(i \theta\left(t_{k}+t\right)\right)\right|
$$

where $K$ is the number of trials, $t$ is the time with respect to the onset of a particular element tone (ranging from 


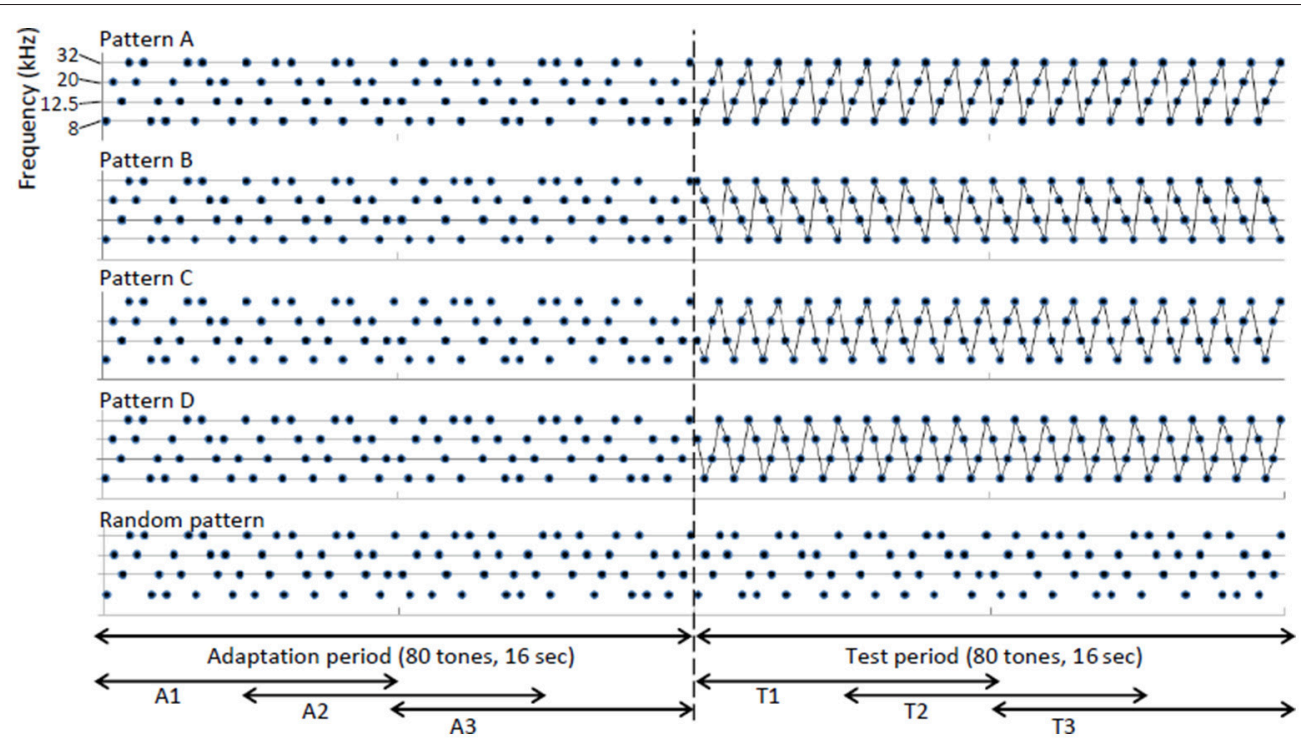

FIGURE 1 | Design of test stimuli. Each sequence consisted of 8, 12.5, 20, or 32-kHz element tones with an inter-tone interval of $200 \mathrm{~ms}$. Each tone sequence started with an adaptation period of $16 \mathrm{~s}$ ( 80 tones), which was followed by a 16-s test period (80 tones). During the adaptation period, each element tone appeared in a pseudo-random order. During the test period, a particular order of four element tones was repeated in four test sequences (patterns A-D), while each element tone continued to appear in a pseudo-random order during the control sequence (random pattern). The adaptation and test periods were divided into three sub-periods of $8 \mathrm{~s}$ with overlaps of $4 \mathrm{~s}$ (A1-A3 and T1-T3).

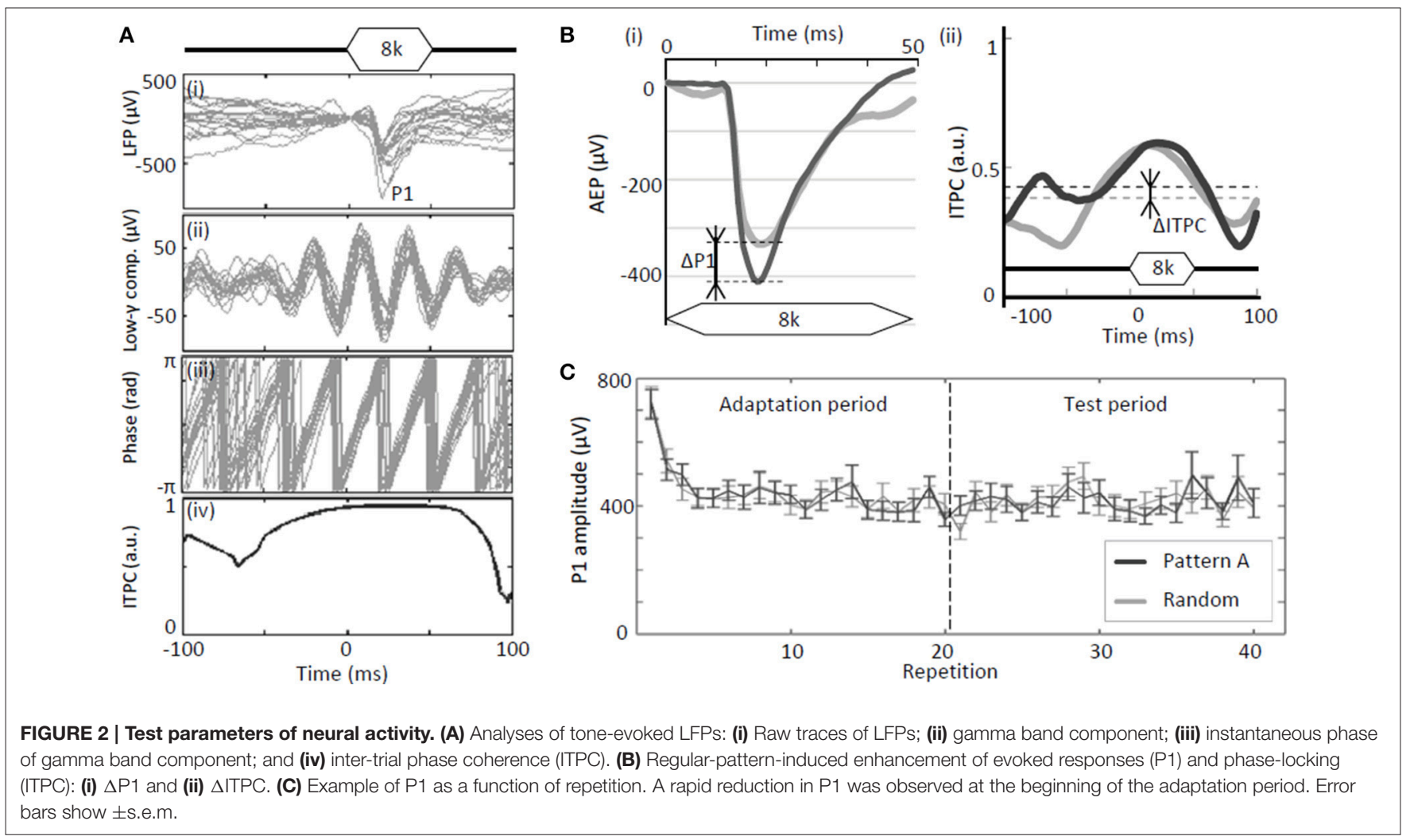

-100 to $100 \mathrm{~ms}$ in the present analysis), $i$ is an imaginary number, $\theta$ is the instantaneous phase, and $t_{k}$ is the time of stimulus onset of a given element tone in the $k^{\text {th }}$ trial.
ITPC is the subtraction of circular variance from 1 (Allen and Johnson, 1991; Marella and Ermentrout, 2008). ITPC ranges between 0 and 1 and nears 1 if the phase at a specific time 
varies slightly across trials. Otherwise, ITPC is close to 0 . The average ITPC values from $t=-100 \mathrm{~ms}$ to $t=100$ ms were used to evaluate the phase modulation by each tone:

$$
\overline{I T P C}=\frac{1}{N} \sum_{c h}\left\{\frac{1}{T} \sum_{t} \operatorname{ITPC}(t)\right\}
$$

Similar to P1, the differences between $\overline{I T P C}$ during the test period for each regular sequence (patterns A-D) and $\overline{I T P C}$ in the control sequence (random pattern) were defined as $\triangle I T P C$ (Figure 2Bii). A positive $\triangle I T P C$ indicates that a regular tone sequence leads to stronger phase locking than a random sequence.

To evaluate the spatial spread of the regularity-induced modulation of P1 amplitude and ITPC, $\triangle \mathrm{P} 1$ and $\triangle$ ITPC at each site were quantified as a function of distance from a P1 local focus based on either a physical distance $(\mathrm{mm})$ or a tonotopic distance (octave). Tonotopic distance was defined as the difference in CF between a pair of given sites.

To test the significance of regularity-induced modulation, two-way analysis of variance (ANOVA) was used considering the following null hypotheses: (i) $\triangle$ ITPCs are equal between the adaptation and test periods (main-period factor; A1-A3 vs. T1T3) and that (ii) $\triangle$ ITPCs are equal among the sub-periods (subperiod factor) either during the adaptation periods (A1 vs. A2 vs. A3) or the test periods (T1 vs. T2 vs. T3). The main-period factor tested whether a transition from random to regular tone sequences modulated $\triangle \mathrm{ITPC}$. If so, the sub-period factor tested whether $\triangle$ ITPC evolved over time.

\section{RESULTS}

For both regular-tone and random-tone sequences, P1 amplitudes of tone-evoked responses rapidly decayed during the initial several seconds owing to adaptation to the tones; however, it relatively stabilized thereafter (Figure 2C). Therefore, our major interests are the P1s and ITPCs of these fully adapted, stable responses during the test period, i.e., repetitions 21-40 (8-16s after the onset of the tone sequences).

\section{Tonotopic Representations of P1 and ITPC}

The test region exhibited a clear tonotopic map, or a place code for frequency. These maps are routinely characterized using MUAs (Figure 3Ai). The primary auditory cortex (AI) in the most dorsal auditory field contains a complete high-tolow tonotopic gradient running along the rostral-to-caudal axis. The anterior auditory field (AAF) has a complete high-to-low tonotopic gradient along the posterodorsal-to-anteroventral axis. The ventral auditory field (VAF) and the suprarhinal auditory field (SRAF) about the ventral border of the AI and the posterior border of the AAF. The posterior auditory field (PAF) is defined as posterior to the A1. Finally, the anterior ventral auditory field (AVAF) is defined on the basis of tonotopic discontinuity at the ventral border of the AAF and the anteroventral border of the SRAF (Horikawa et al., 1988; Rutkowski et al., 2003; Kalatsky et al., 2005; Polley et al., 2007; Higgins et al., 2010; Storace et al.,
2011; Takahashi et al., 2011; Funamizu et al., 2013). Consistent with this map, the P1 exhibited tonotopic representation, where a low-frequency tone $(8 \mathrm{kHz})$ led to several activation foci appearing at the fringe, while a high-frequency tone $(32 \mathrm{kHz})$ appeared in the middle (Figure 3Aii).

The tonotopic patterns of P1 were qualitatively similar during the regular and random sequences (Figures 3Bi,ii). However, the differential patterns between regular and random sequences, i.e., $\Delta \mathrm{P} 1$, revealed that $\Delta \mathrm{P} 1$ was dependent on the tones of the test elements. Here, $\Delta \mathrm{P} 1$ tended to be positive in response to $8-\mathrm{kHz}$ tones and was negative in response to $32-\mathrm{kHz}$ tones (Figure 3Biii). ITPC was highly correlated with P1 (Pearson's coefficients between P1 and ITPC map: $8 \mathrm{kHz}, R=0.83 ; 32$ $\mathrm{kHz}, R=0.81$; Figures $3 \mathrm{~B}, \mathrm{C}$ ). This indicates that ITPC is also tonotopically organized. The differential patterns of ITPC in response to regular and random sequences, i.e., $\triangle \mathrm{ITPC}$, were also dependent on the tones of the test elements.

\section{Neural Signature of Regularity}

We investigated whether the regularity of a tone sequence modulated P1 independently of the test pattern. The $\Delta \mathrm{P} 1$ of the largest $\mathrm{P} 1$ in the activation map was quantified as a function of the test stimuli. For identical tones, $\Delta \mathrm{P} 1$ depended on test patterns. It was significantly positive in response to some patterns, but was negative to in response to others (two-sided $t$-test, $p<$ 0.05 ; Figure $4 \mathrm{~A}$ ). This pattern-dependent $\Delta \mathrm{P} 1$ was likely caused by forward masking because $\Delta \mathrm{P} 1$ was significantly positively correlated with the separations between the provided test frequencies and those of the preceding tones $(\Delta \mathrm{F})$ (Figure $4 \mathrm{~B}$ ). In other words, the $\mathrm{P} 1$ in response to a given tone was suppressed when a subset of the neural population preferring the test tone was activated by the preceding tone with an adjacent frequency. This result suggests that P1 amplitude is not a candidate for the pattern-independent neural representation of regular tone sequences.

We also tested whether $\triangle$ ITPC at a given frequency band may be the neural correlate for the extraction of regular tone sequences (Figure 5). The average of $\triangle$ ITPC over the auditory cortex was quantified, as ITPC was less localized in the auditory cortex than P1 was (Figures 3, 6). Similar to $\triangle \mathrm{P} 1, \triangle \mathrm{ITPC}$ in response to identical tones depended on the test patterns. However, we found pattern-independent modulation in the lowgamma band, where almost all of the test tones were significantly positive (two-sided $t$-test, $p<0.05$; Figure 5C). This suggests that the low-gamma ITPC increases in the presence of any kind of regular tone sequence.

\section{Spatial Spread of Neural Signature}

To characterize the spatial spread of the neural signature of regularity, we compared $\triangle \mathrm{P} 1$ and $\triangle \mathrm{ITPC}$ in terms of spatial profile. $\Delta \mathrm{P} 1$ exhibited local maxima around the activation foci of $\mathrm{P} 1$ and monotonically decreased with distance from the focus with a halfwidth of $<0.4 \mathrm{~mm}$ (Figure 6A). However, $\triangle I T P C$ did not have a local maximum around the P1 focus. It had a non-monotonic function with respect to the distance from the foci of the local maxima depending on the oscillation band (Figure 6B): alpha, 0.2-0.6 mm (with a halfwidth of $<$ 
A

(i)

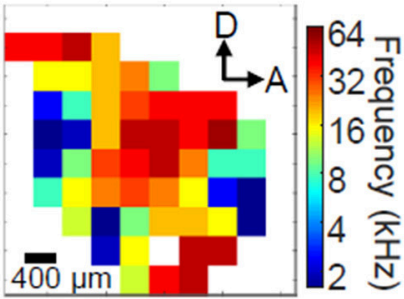

B P1

$8 \mathrm{k}$

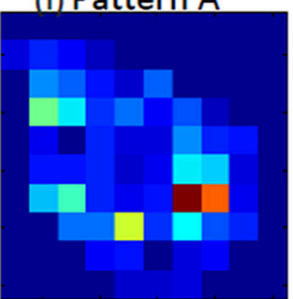

$2 k$

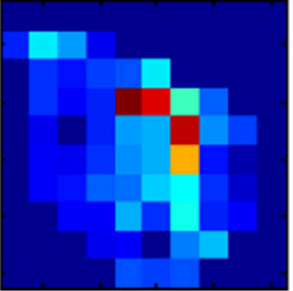

C ITPC (i) Pattern A
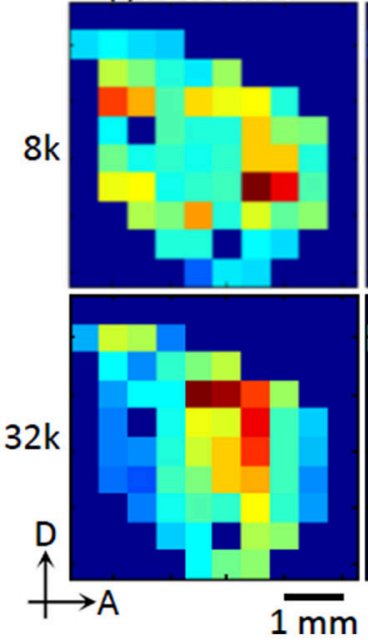

(ii) Random (ii)

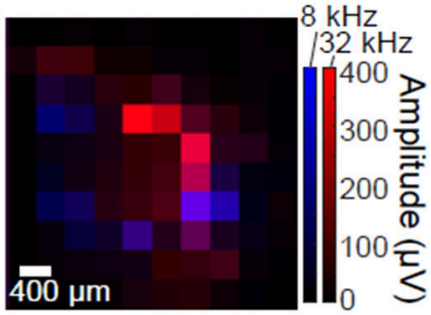

(iii) $\triangle \mathrm{P} 1$
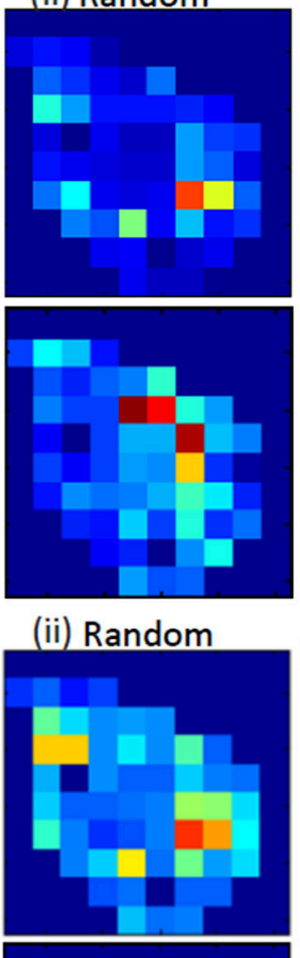

ITPC (au)

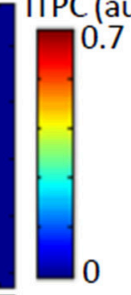

(iii) $\triangle$ ITPC

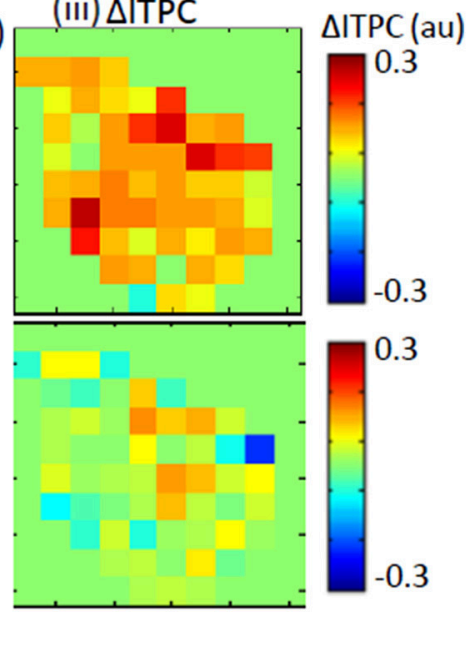

FIGURE 3 | Representative map of tone-evoked response amplitudes and phase-locking. (A) Tonotopic map obtained from MUA (i). Tonotopic activation of P1 (ii). (B) P1 amplitude. (C) ITPC at low gamma band. (i) Regular sequence (Pattern A); (ii) random sequence; and (iii) enhancement of P1 and ITPC during regular sequences compared to those random sequences ( $\triangle \mathrm{P} 1$ and $\Delta \mathrm{ITPC}$ ). The test frequencies used to examine these maps were 8 and $32 \mathrm{kHz}$.

$2.4 \mathrm{~mm}$ ); beta, $0.6-1.0 \mathrm{~mm}$ (halfwidth, $<2.8 \mathrm{~mm}$ ); low-gamma, 1.0-1.4 mm (halfwidth, $<2.4 \mathrm{~mm}$ ); and high-gamma, $0.6-1.0 \mathrm{~mm}$ (halfwidth, $<1.6 \mathrm{~mm}$ ). The spatial decays of $\triangle \mathrm{ITPC}$, where $\triangle$ ITPC became significantly smaller than the maximum $\triangle I T P C$, were $2.2-2.6 \mathrm{~mm}$ in the alpha band, $1.4-1.8 \mathrm{~mm}$ in the beta band, $1.0-1.4 \mathrm{~mm}$ in the low-gamma band, and $1.4-1.8 \mathrm{~mm}$ in the high-gamma band. These spatial decays were significantly larger than those of $\Delta \mathrm{P} 1(0.6-1.0 \mathrm{~mm})$ (Mann-Whitney $U$-test with Bonferroni correction, $p<0.05$ ). These results suggest that $\triangle$ ITPC spreads more widely over the auditory cortex than $\Delta \mathrm{P} 1$.
We further characterized the spatial spread of low-gamma ITPC and $\triangle I T P C$, because these phase-locking features are candidates for the neural signature representing sound pattern regularity (Figure 5). The low-gamma ITPC significantly depended on the separation of the test frequency from the $\mathrm{CF}$ at a given recording site (i.e., tonotopic distance or $\triangle \mathrm{CF}$ ) during both the adaptation and test periods [Figure 6C; oneway ANOVA: $F_{(8,31,796)}=405.38, p<10^{-4}$ for the random sequence; $F_{(8,7975)}=136.21, p<10^{-4}$ for the regular sequence]. Consistent with the spatially averaged data (Figure 5), the 


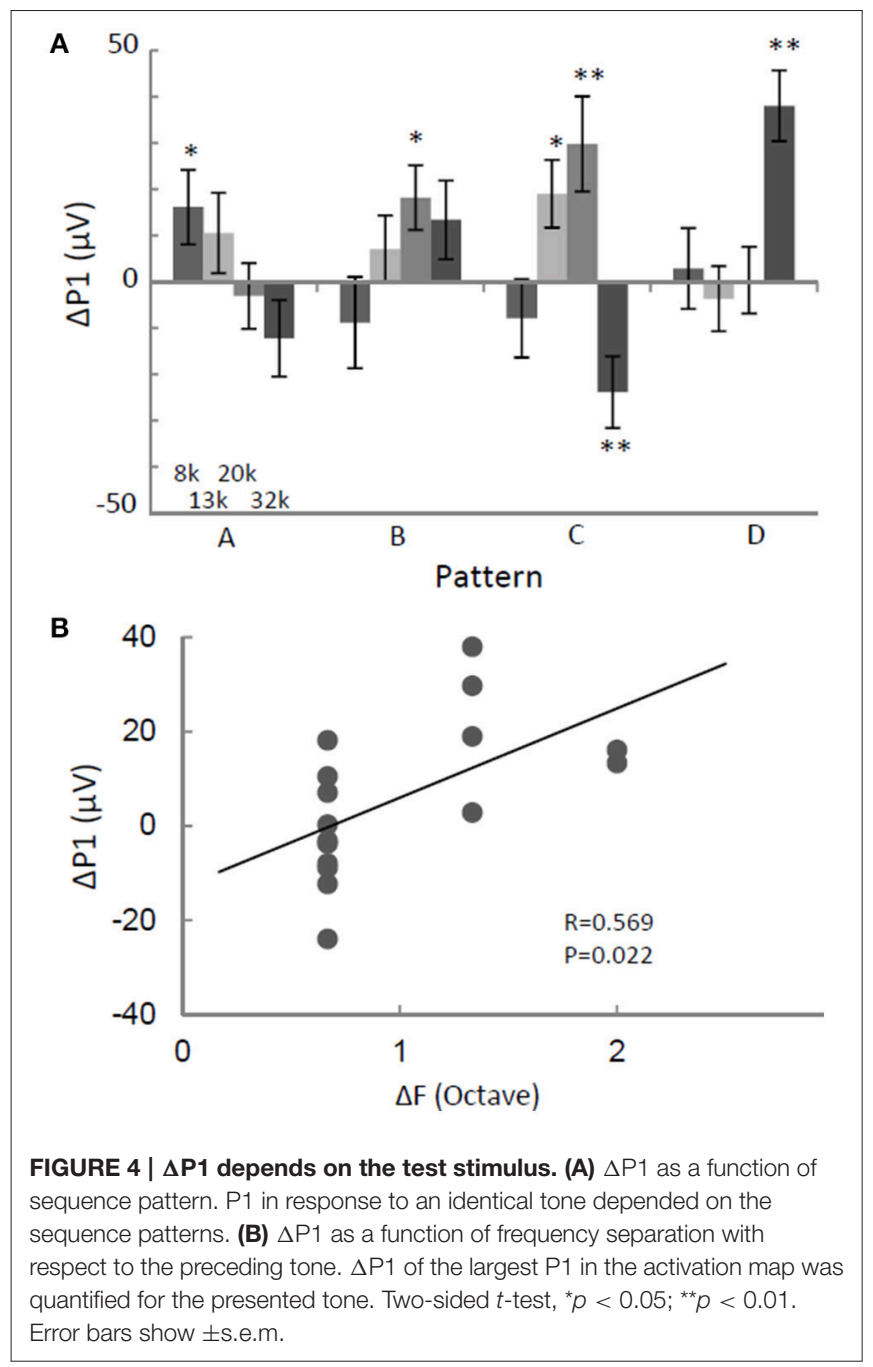

low-gamma ITPCs in the regular patterns were higher than that in the random patterns at any site in the auditory cortex, i.e., irrespective of $\triangle \mathrm{CF}$. Closer inspection revealed that the $\triangle \mathrm{ITPC}$ at the $\mathrm{CF}$ sites of the test tones $(\Delta \mathrm{CF}=0)$ were significantly lower than those at the surrounding sites [Figure 6D; $\triangle \mathrm{CF}$ approximately \pm 0.67 octave; one-way ANOVA, $F_{(8,31,796)}=$ $33.3, p<10^{-4}$; post-hoc two-sided $t$-test, $\left.p<0.01\right]$. This suggests that tone repetition exerts a more considerable impact on phase locking at frequencies surrounding the CF rather than the CFs of the test tones.

\section{Evolution of Phase Locking Modulation}

The neuronal signature of regularity might be produced in either a bottom-up or a top-down manner. A bottom-up signature probably evolves immediately upon the switch from a random to a regular sequence, whereas a top-down signature probably evolves gradually with time after the switch. Thus, we investigated the time courses of $\triangle$ ITPC and found that $\triangle$ ITPC evolves in a band-specific manner (Figure 7). Significant main-period effects were observed in the alpha $\left[F_{(1,90)}=12.92, p=0.00052\right]$ and

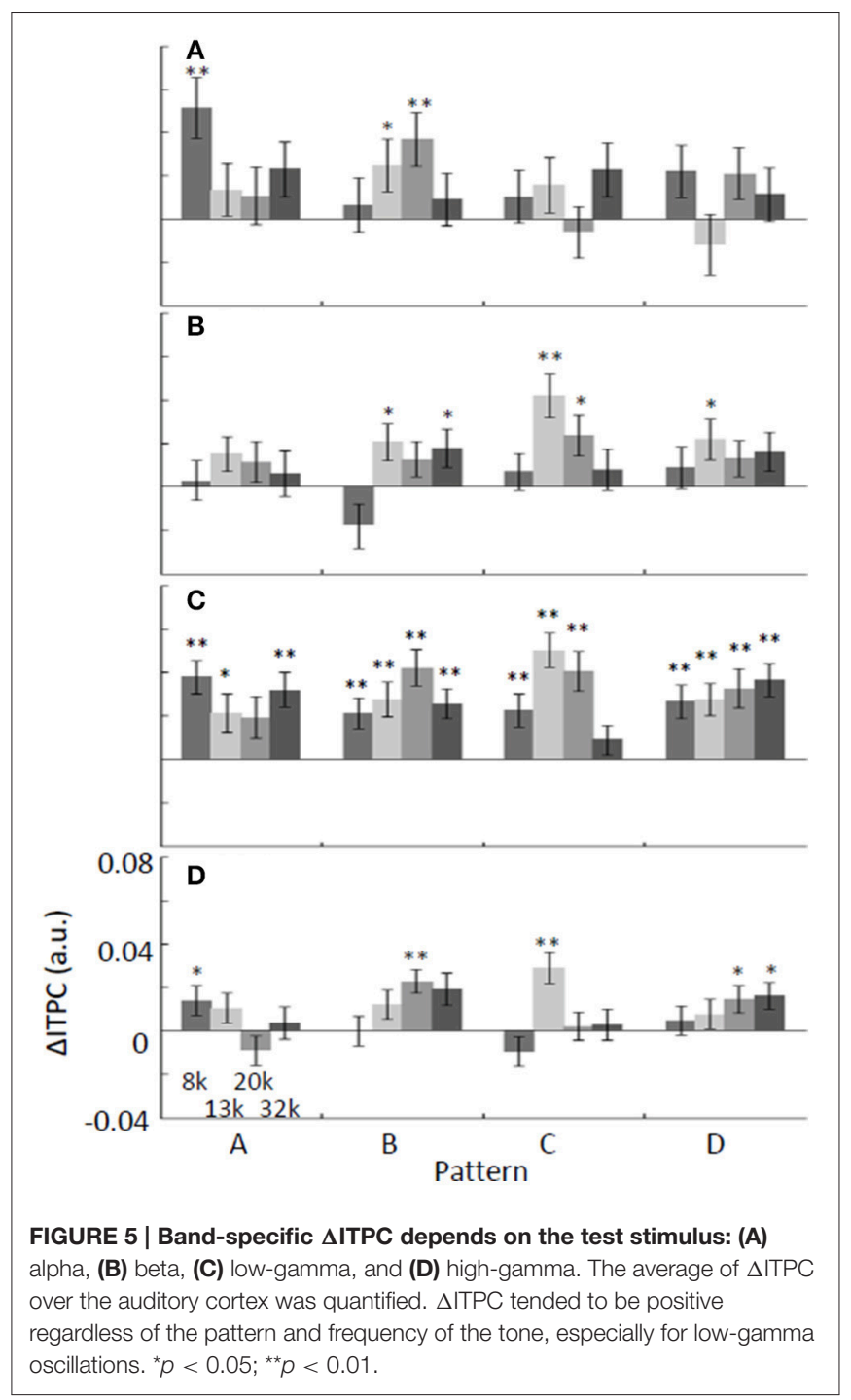

low-gamma $\left[F_{(1,90)}=103.26, p<10^{-4}\right]$ bands without subperiod effects or interactions between the two factors. More interestingly, the beta band $\triangle$ ITPC exhibited a significant mainperiod effect $\left[F_{(1,90)}=8.2, p=0.0052\right]$, a sub-period effect $\left[F_{(2,90)}=6.21, p=0.0030\right]$, and an interaction between these two factors $\left[F_{(2,90)}=14.07, p<10^{-4}\right]$. The sub-period effect in the beta band was significant only during the test period [oneway ANOVA, $\left.F_{(2,45)}=13.89, p<10^{-4}\right]$. No significant effect was observed in the high-gamma band. Along with the results shown in Figure 5, regular-tone sequences tended to induce phase locking in the alpha, beta, and low-gamma bands. The phase locking in the beta band evolved with time.

\section{Amplitude Modulation vs. Phase Locking Modulation}

We determined whether and how low-gamma $\triangle$ ITPC depended on the amplitudes of tone-evoked responses. There was no significant correlation between low-gamma $\triangle$ ITPC and P1 


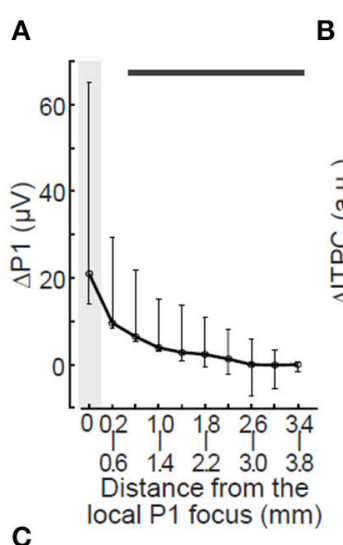

C
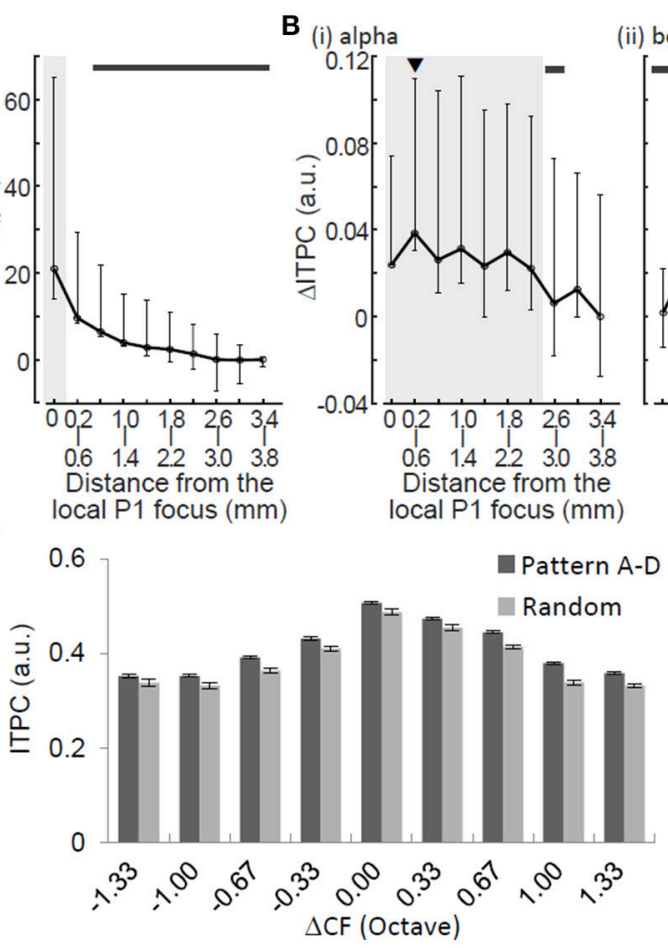

(ii) beta
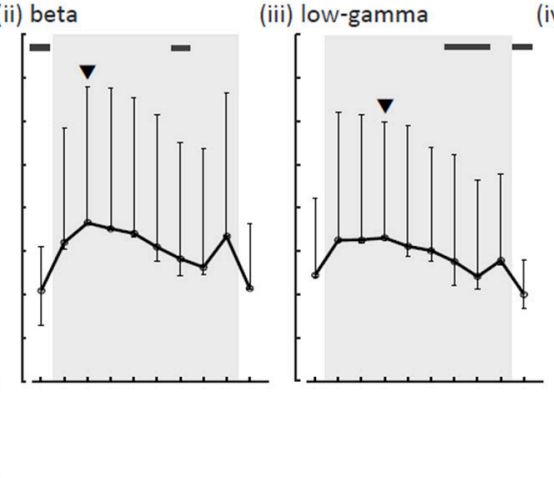

(iv) high-gamma

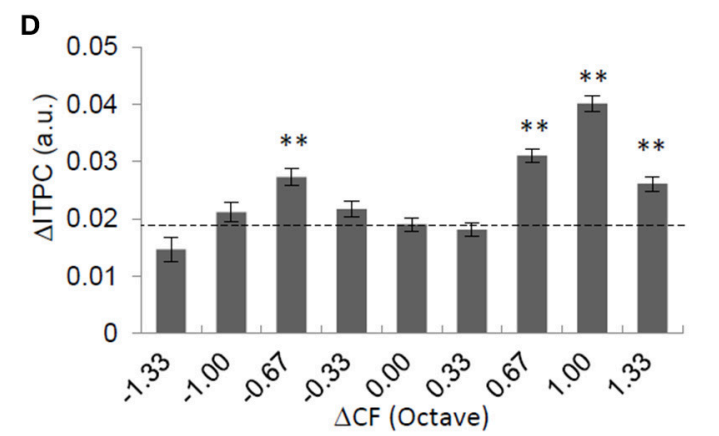

FIGURE 6 | Spatial characterizations of $\Delta$ P1 and $\mathbf{\Delta I T P C . ~} \Delta \mathrm{P} 1$ (A) and $\triangle$ ITPC (B) as functions of physical distance from the P1 local focus. Median and error bars indicating 25 and $75 \%$ quartile deviations are provided. Black arrowheads in (B) indicate the local maxima of $\Delta$ ITPC. Shaded areas indicate the ranges of half-widths in relation to the local maxima. Thick horizontal bars indicate that $\triangle \mathrm{P} 1$ or $\triangle$ ITPC at a given distance are significantly smaller than the local maxima (Mann-Whitney $U$-test with Bonferroni correction, $p<0.05)$. ITPC (C) and $\Delta$ ITPC (D) in the low-gamma band as a function of tonotopic distance ( $\Delta$ CF in octave). Two-sided $t$-test, ${ }^{\star *} p<0.01$.

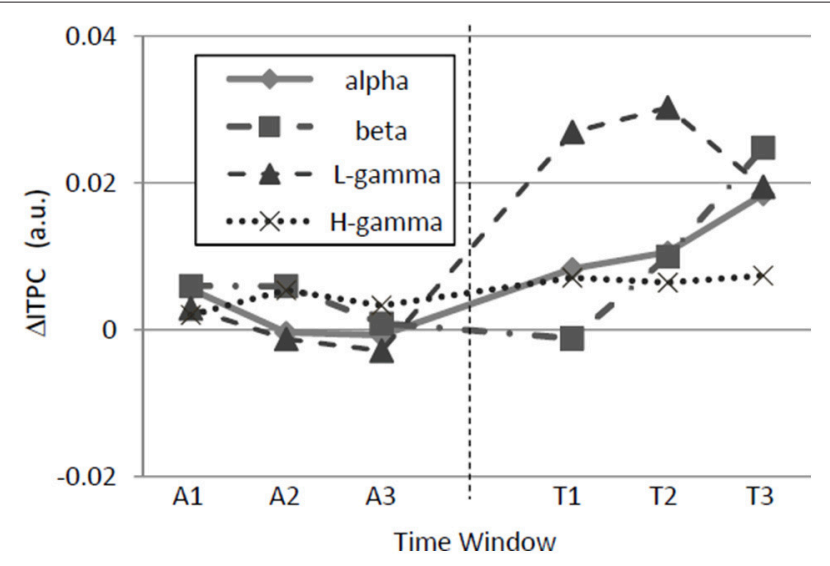

FIGURE 7 | Time variations of band-specific $\Delta$ ITPCs. In the alpha, beta, low-gamma, and high-gamma bands, $\triangle$ ITPC was significantly higher during the test period than during the adaptation period. Additionally, in the beta band, $\triangle$ ITPC evolved over time during the test period. Similar to Figure 5, $\triangle$ ITPC is the averaged value across the auditory cortex.

(Figure 8; Pearson's coefficient $R_{x y}=0.274$; two-sided $t$-test, $p=$ 0.29 ). This indicates that large auditory evoked responses do not lead to increased phase locking upon the transition from random to regular tone sequences. However, low-gamma $\triangle$ ITPC was significantly positively correlated with $\Delta \mathrm{P} 1$ (Pearson's coefficient $R_{x y}=0.727$; two-sided $t$-test, $p=0.0014$ ). This suggests that increases in $\mathrm{P} 1$ in response to particular tones trigger tight phase locking upon the transition from a random to a regular sequence and that they enhance overall phase locking to tones in response to repeated sequences.

\section{DISCUSSION}

\section{Summary of Findings}

Neuronal responses specific to regular tone sequences in the auditory cortex were characterized by comparing tone-evoked responses to regular sequences with those in responses to random sequences. The amplitudes of tone-evoked responses (P1) depended on test patterns such that P1 increased when the frequency separation between a given tone and the prior tone was large during regular sequences (Figure 4). Therefore, P1 amplitude is likely to encode the local order of the test patterns rather than serve as a global sign of sound repetition. In contrast to P1 amplitude, band-specific phase locking to tones (ITPC) tended to be larger during regular tone sequences than during the random sequences (Figure 5). This enhancement in phaselocking ( $\triangle \mathrm{ITPC}$ ) was observed widely over the whole auditory cortex area (Figures 3, 6). These results support the idea that widespread phase locking to regular tones plays a more important 


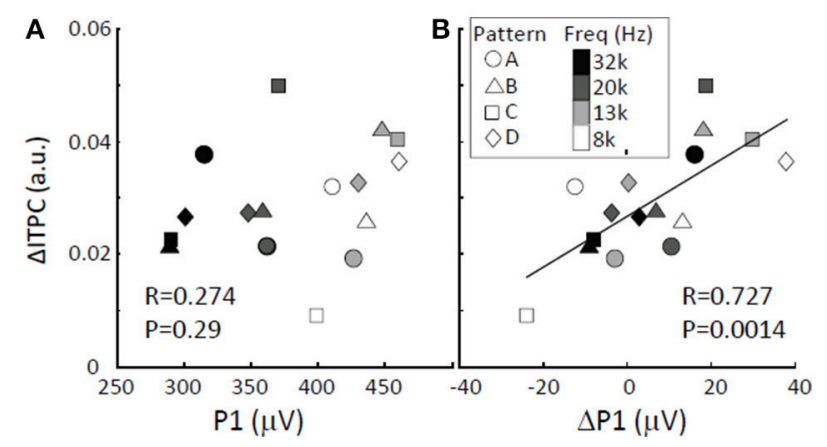

FIGURE 8 | $\triangle$ ITPC vs. LFP amplitude. (A) No correlation was found between low-gamma $\triangle$ ITPC and P1. (B) Positive correlation between low-gamma $\triangle$ ITPC and $\triangle \mathrm{P} 1$. The largest $\mathrm{P} 1$ and the corresponding $\Delta \mathrm{P} 1$ were obtained from the activation map in response to the presented tone, while the $\triangle$ ITPC is the averaged value across the auditory cortex.

role in the neural processes involved in detecting regularity of tone sequences rather than those responsible for the detection of the local order of tone sequences. We found that phase locking evolves in a band-specific manner. Gamma-band phase locking increases immediately after the transition from random to regular sequences, while beta-band phase locking gradually evolves with time after the transition (Figure 7). Thus, each band is likely to play a different role in the neuronal detection of repeated tone sequences at different points of temporal evolution. We also observed that pattern-dependent enhancements of phase locking were positively correlated with those of $\mathrm{P} 1$. This suggests that increases in $\mathrm{P} 1$ in response to particular tones trigger tight phase locking (Figure 8).

\section{P1 Amplitude vs. ITPC}

$\mathrm{P} 1$, which is a major short latency response component, exhibited rapid adaptation to repeated tone sequences (Figure 2C). Since the recording sites were located at the depth of the thalamo-recipient layer in the cortex, the major mechanisms of this adaptation might involve fatiguing of thalamic inputs (Antunes and Malmierca, 2011, 2014) and thalamo-cortical synaptic depression (Wehr and Zador, 2005; Taaseh et al., 2011; Hershenhoren et al., 2014; Nelken, 2014). The degree of depression depends on the frequency difference between the present tone and the preceding tone. This is known as forward masking (Brosch and Schreiner, 1997; Noda et al., 2013b).

Stimulus phase locking is partly caused by highly reliable tone-evoked responses (Edwards et al., 2009). In fact, the spatial distributions of ITPC were tonotopically organized (Figure 3C), similar to the P1 distributions. The increments of ITPC in response to a regular tone sequence compared to a random tone sequence also showed tight positive correlations with those of the P1 amplitude (Figure 8).

We characterized the P1 amplitude as the average of the peak LFP values in each trial. Therefore, instead of the peak of the trial-averaged evoked response, P1 amplitude is theoretically independent of temporal and phase information in a sharp LFP transient. The time window to quantify phase-locking (200 ms) was also much longer than the duration of the P1 component (several tens of milliseconds). Thus, ITPC is essentially different from P1 amplitude. These test parameters differ in several respects. First, the spatial distributions of the ITPC increment were less local and were more widely distributed than those of P1 amplitude. Second, the increments of ITPC were always positive even though no P1 increment was observed in some test conditions. Third, the coefficient of variation of the P1 amplitude in response to various tone patterns was not correlated with the corresponding ITPC. This indicates that amplitude differed from phase in terms of inter-trial variability (Supplementary Figure 1). Fourth, the regularity-induced evolution was observed only in phase locking but not in P1 amplitude. Fifth, a rapid fall-off was clearly observed in $\mathrm{P} 1$ amplitude within a few repetitions of the tones. However, there was no such fall-off in ITPC (Noda et al., 2013a). The differences between P1 amplitude and ITPC are likely caused by the phase reset of the ongoing LFP without evoking P1 responses (Makeig et al., 2002; Sauseng et al., 2007; Barry, 2009; Kayser, 2009; Lakatos et al., 2009).

\section{Spatial Spread of Phase Locking}

The wide distribution of phase-locking compared to P1 amplitude suggests that the neural mechanism of phase locking (e.g., phase reset) works with a low level of thalamo-cortical input, while the local distribution of the P1 amplitude is shaped by feedforward side-band inhibition (Wang and Salvi, 2002; Zhang et al., 2003; Llinás et al., 2005). Additionally, phase locking may be enhanced over a wide range by cortico-cortical horizontal connections via the axonal collaterals of pyramidal cells or interneurons. This may lead to synchronization of neuronal populations and the transmission of signals in a bandspecific manner (Traub et al., 1996; Buzsáki and Wang, 2012). Stable global oscillations with high phase locking are established in the presence of regular tone sequences because a number of oscillatory activities are simultaneously enabled by reliable thalamo-cortical inputs.

An increase in ITPC in response to regular tone sequences was prominent in CF regions $0.67-1.00$ octave away from regions with the $\mathrm{CF}$ of the test frequency (Figure 6D). This spatial spread of phase locking may be enabled by short-range intracortical connections (Happel et al., 2010) and contributes to the grouping of tones (e.g., $8-12.5-20 \mathrm{kHz}$ and $12.5-20-$ $32 \mathrm{kHz}$ ). From a computational perspective, such grouping through temporal phase-locking makes the encoding of regular patterns more robust than that of random patterns without consuming additional energy (Buzsaki and Draguhn, 2004; Fell and Axmacher, 2011; Yokota et al., 2015).

\section{Band-Specific Phase Locking}

We found that gamma-band phase locking immediately increases in the first sub-period ( $0-8 \mathrm{~s})$ of the regular tone sequence and maintains a high value throughout the remaining sub-periods of the sequence (4-12 and 8-16 s) (Figure 7). However, beta band phase locking gradually increases during the second sub-period (4-12 s) and reaches its maximum during the last sub-period (8$16 \mathrm{~s})$. These band-specific temporal evolutions suggest that each band plays a different role in our experiments. 
Gamma oscillations have long been known to play a role in the temporal binding of sensory features into a coherent percept (Gray et al., 1989; Engel et al., 2001). According to this traditional viewpoint, the gamma oscillations present during the presentation of regular tone sequences in our study may indicate that repeated tones are temporally bound as an auditory object. Additionally, the rapid evolution of gamma oscillations suggests that this binding process is an automatic bottomup process that is a "primitive" auditory feature. This gamma band synchronization is likely to occur locally in a feedforward manner.

Compared to the gamma rhythm, the slower rhythms in the alpha to beta bands are used to organize longer-range spatial and temporal synchronous activity (Kopell et al., 2000; Siegel et al., 2012; Lee et al., 2013; Fries, 2015). For example, beta band modulation is associated with motor control not only in the cortex (Engel and Fries, 2010) but also in the thalamus (Paradiso et al., 2004) and basal ganglia (Cassidy et al., 2002; Foffani et al., 2005). In addition, beta activity in the auditory cortex is associated with beat processing in concert with the motor and association cortices and the cerebellum (Fujioka et al., 2012, 2015). The beta band activities in the cortex emerge from the deep cortical layers (Wang, 2010; Markov et al., 2013) and may transmit signals in a feedback or top-down manner (von Stein and Sarnthein, 2000; Buschman and Miller, 2007; Chandrasekaran and Ghazanfar, 2009; de Graaf et al., 2013).

Gamma-band and beta-band phase locking may be differentially involved in predictive timing. According to the predictive coding hypothesis, gamma oscillations convey bottom-up prediction errors in sensory information (e.g., violation of sound expectation), whereas beta oscillations provide top-down prediction (Friston, 2005, 2009; Arnal and Giraud, 2012; Siegel et al., 2012; Malmierca et al., 2015). During the random sequence, where the prediction of the upcoming tone is impossible, no prediction error can be produced. Therefore, there is no predictive timing during the random sequence. Immediately after the transition from the random to the regular sequence, temporal regularity should enable predictive coding, possibly with a transition response (i.e., the largest error signal) appearing within a few cycles after the transition (Chait et al., 2008). Such immediate initiation of predictive coding should simultaneously enable predictive timing, which is associated with the rapid evolution of gamma-band phase locking in this present study. Thereafter, stable prediction may then become gradually available, as indexed by the gradual evolution of beta-band phase locking. Thus, our results may provide additional evidence that gamma and beta-band phase locking play compensatory roles in predictive timing.

Our results suggest that predictive timing is active under anesthesia. We have characterized phase locking in an early component of evoked potential, which is less vulnerable to conscious states than late response components (Del Cul et al., 2007). There are also several pre-attentive mechanisms in predictive coding. These include stimulus-specific adaptation and mismatch negativity (Friston, 2005; Peretz et al., 2009; Winkler et al., 2009; Wacongne et al., 2011, 2012; Bendixen et al., 2012; Shiramatsu et al., 2013; Malmierca et al., 2015). However, further experiments are still required to elucidate the effects of anesthesia on our findings, as isoflurane has substantial impacts on oscillatory activities (Imas et al., 2005; Noda and Takahashi, 2015). Anesthesia also induces loss of consciousness (Alkire et al., 2008; Raz et al., 2014), which weakens top-down information transfer. The powerful top-down control exerted by attention during waking may alter the neural signature of regularity. Awake-state behavioral experiments will promote a better understanding of how top-down and bottom-up mechanisms contribute to the perception of tone sequence regularity. In these experiments, lengthening the duration between the tones would allow us to investigate whether tone sequence regularity modulates tone-induced oscillatory components (Tallon-Baudry et al., 2004; Tallon-Baudry and Bertrand, 2007). It would be also intriguing to comprehensively characterize tone pattern-specific phase locking across different frequency bands. Furthermore, causal links between neural activity in the auditory cortex and other regions is of great interest to us and can be used to address whether and how beta and gamma phase locking are associated with top-down and bottom-up information flow, respectively.

In summary, regular-tone sequences spatio-temporally modulate the stimulus phase locking of neural oscillations in the auditory cortex with global scales, whereas the amplitudes of tone-evoked responses to each component encode the sequence information with local scales, i.e., the local order of tones. Additionally, band-specific temporal evolution of oscillatory phase locking may support the recent theories regarding predictive timing that gammaband phase locking encodes bottom-up prediction errors while beta-band phase locking is involved in top-down prediction.

\section{AUTHOR CONTRIBUTIONS}

TN, TA, and HT designed experiments. TN, TA, and HT analyzed data. TN, TA, and TS conducted physiological experiments. TN, TA, and HT evaluated results. TN, TA, and HT made all figures. TN, TA, and HT wrote the manuscript.

\section{FUNDING}

This work was partially supported by KAKENHI Grant (25135710, 26242040, 16H01604).

\section{ACKNOWLEDGMENTS}

The authors are grateful to helpful comments from Dr. Makio Kashino and Dr. Shigeto Furukawa at NTT Communication Science Laboratories, NTT Corporation, Atsugi, Japan. TA is now with Hitachi, Ltd., Tokyo, Japan.

\section{SUPPLEMENTARY MATERIAL}

The Supplementary Material for this article can be found online at: http://journal.frontiersin.org/article/10.3389/fncir. 2017.00002/full\#supplementary-material 


\section{REFERENCES}

Abe, K., and Watanabe, D. (2011). Songbirds possess the spontaneous ability to discriminate syntactic rules. Nat. Neurosci. 14, 1067-1074. doi: 10.1038/nn.2869

Alkire, M. T., Hudetz, A. G., and Tononi, G. (2008). Consciousness and anesthesia. Science 322, 876-880. doi: 10.1126/science.1149213

Allen, F. H., and Johnson, O. (1991). Automated conformational analysis from crystallographic data. 4. Statistical descriptors for a distribution of torsion angles. Acta Cryst. B47, 62-67. doi: 10.1107/S0108768190010382

Antunes, F. M., and Malmierca, M. S. (2011). Effect of auditory cortex deactivation on stimulus-specific adaptation in the medial geniculate body. J. Neurosci. 31, 17306-17316. doi: 10.1523/JNEUROSCI.1915-11.2011

Antunes, F. M., and Malmierca, M. S. (2014). An overview of stimulusspecific adaptation in the auditory thalamus. Brain Topogr. 27, 480-499. doi: 10.1007/s10548-013-0342-6

Arnal, L. H., and Giraud, A. L. (2012). Cortical oscillations and sensory predictions. Trends Cogn. Sci. 16, 390-398. doi: 10.1016/j.tics.2012.05.003

Barry, R. J. (2009). Evoked activity and EEG phase resetting in the genesis of auditory Go/NoGo ERPs. Biol. Psychol. 80, 292-299. doi: 10.1016/j.biopsycho.2008.10.009

Bendixen, A., Roeber, U., and Schröger, E. (2007). Regularity extraction and application in dynamic auditory stimulus sequences. J. Cogn. Neurosci. 19, 1664-1677. doi: 10.1162/jocn.2007.19.10.1664

Bendixen, A., Schröger, E., Ritter, W., and Winkler, I. (2012). Regularity extraction from non-adjacent sounds. Front. Psychol. 3:143. doi: 10.3389/fpsyg.2012.00143

Brosch, M., and Schreiner, C. (1997). Time course of forward masking tuning curves in cat primary auditory cortex. J. Neurophysiol. 77, 923-943.

Busch, N. A., Dubois, J., and VanRullen, R. (2009). The phase of ongoing EEG oscillations predicts visual perception. J. Neurosci. 29, 7869-7876. doi: 10.1523/JNEUROSCI.0113-09.2009

Busch, N. A., and VanRullen, R. (2010). Spontaneous EEG oscillations reveal periodic sampling of visual attention. Proc. Nati. Acad. Sci. U.S.A. 107, 16048-16053. doi: 10.1073/pnas.1004801107

Buschman, T. J., and Miller, E. K. (2007). Top-down versus bottom-up control of attention in the prefrontal and posterior parietal cortices. Science 315, 1860-1862. doi: 10.1126/science.1138071

Buzsáki, G., and Draguhn, A. (2004). Neuronal oscillations in cortical networks. Science 304, 1926-1929. doi: 10.1126/science.1099745

Buzsáki, G., and Wang, X. J. (2012). Mechanisms of gamma oscillations. Annu. Rev. Neurosci. 35, 203-225. doi: 10.1146/annurev-neuro-062111-150444

Cassidy, M., Mazzone, P., Oliviero, A., Insola, A., Tonali, P., Di Lazzaro, V., et al. (2002). Movement-related changes in synchronization in the human basal ganglia. Brain 125, 1235-1246. doi: 10.1093/brain/awf135

Chait, M., Poeppel, D., and Simon, J. Z. (2008). Auditory temporal edge detection in human auditory cortex. Brain Res. 1213, 78-90. doi: 10.1016/j.brainres.2008.03.050

Chandrasekaran, C., and Ghazanfar, A. A. (2009). Different neural frequency bands integrate faces and voices differently in the superior temporal sulcus. J. Neurophysiol. 101, 773-788. doi: 10.1152/jn.90843.2008

de Graaf, T. A., Gross, J., Paterson, G., Rusch, T., Sack, A. T., and Thut, G. (2013). Alpha-band rhythms in visual task performance: phase-locking by rhythmic sensory stimulation. PLoS ONE 8:e60035. doi: 10.1371/journal.pone.00 60035

de la Mora, D. M., and Toro, J. M. (2013). Rule learning over consonants and vowels in a non-human animal. Cognition 126, 307-312. doi: 10.1016/j.cognition.2012.09.015

Del Cul, A., Baillet, S., and Dehaene, S. (2007). Brain dynamics underlying the nonlinear threshold for access to consciousness. PLoS Biol. 5:e260. doi: 10.1371/journal.pbio.0050260

Edwards, E., Soltani, M., Kim, W., Dalal, S. S., Nagarajan, S. S., Berger, M. S., et al. (2009). Comparison of time-frequency responses and the event-related potential to auditory speech stimuli in human cortex. J. Neurophysiol. 102, 377-386. doi: 10.1152/jn.90954.2008

Engel, A., Fries, P., and Singer, W. (2001). Dynamic prediction, oscillations and synchrony in top-down processing. Nat. Rev. Neurosci. 2, 704-716. doi: $10.1038 / 35094565$
Engel, A. K., and Fries, P. (2010). Beta-band oscillations-signalling the status quo? Curr. Opin. Neurobiol. 20, 156-165. doi: 10.1016/j.conb.2010.02.015

Fell, J., and Axmacher, N. (2011). The role of phase synchronization in memory processes. Nat. Rev. Neurosci. 12, 105-118. doi: 10.1038/nrn2979

Foffani, G., Bianchi, A. M., Baselli, G., and Priori, A. (2005). Movement-related frequency modulation of beta oscillatory activity in the human subthalamic nucleus. J. Physiol. 568, 699-711. doi: 10.1113/jphysiol.2005.089722

Fries, P. (2015). Rhythms for cognition: communication through coherence. Neuron 88, 220-235. doi: 10.1016/j.neuron.2015.09.034

Friston, K. (2005). A theory of cortical responses. Philos. Trans. R. Soc. Lond. B Biol. Sci. 360, 815-836. doi: 10.1098/rstb.2005.1622

Friston, K. (2009). The free-energy principle: a rough guide to the brain? Trends Cogn. Sci. 13, 293-301. doi: 10.1016/j.tics.2009.04.005

Fujioka, T., Ross, B., and Trainor, L. J. (2015). Beta-band oscillations represent auditory beat and its metrical hierarchy in perception and imagery. J. Neurosci. 35, 15187-15198. doi: 10.1523/JNEUROSCI.2397-15.2015

Fujioka, T., Trainor, L. J., Large, E. W., and Ross, B. (2009). Beta and gamma rhythms in human auditory cortex during musical beat processing. Ann. N. Y. Acad. Sci. 1169, 89-92. doi: 10.1111/j.1749-6632.2009.04779.x

Fujioka, T., Trainor, L. J., Large, E. W., and Ross, B. (2012). Internalized timing of isochronous sounds is represented in neuromagnetic beta oscillations. $J$. Neurosci. 32, 1791-1802. doi: 10.1523/JNEUROSCI.4107-11.2012

Funamizu, A., Kanzaki, R., and Takahashi, H. (2013). Pre-Attentive, contextspecific representation of fear memory in the auditory cortex of rat. PLOS ONE 8:e63655. doi: 10.1371/journal.pone.0063655

Giraud, A. L., and Poeppel, D. (2012). Cortical oscillations and speech processing: emerging computational principles and operations. Nat. Neurosci. 15, 511-517. doi: $10.1038 / \mathrm{nn} .3063$

Gray, C. M., König, P., Engel, A. K., and Singer, W. (1989). Oscillatory responses in cat visual cortex exhibit inter-columnar synchronization which reflects global stimulus properties. Nature 338, 334-337. doi: 10.1038/338 $334 \mathrm{a} 0$

Griffiths, T. D., Büchel, C., Frackowiak, R. S. J., and Patterson, R. D. (1998). Analysis of temporal structure in sound by the human brain. Nat. Neurosci. 1, 422-427. doi: 10.1038/1637

Happel, M. F., Jeschke, M., and Ohl, F. W. (2010). Spectral integration in primary auditory cortex attributable to temporally precise convergence of thalamocortical and intracortical input. J. Neurosci. 30, 11114-11127. doi: 10.1523/JNEUROSCI.0689-10.2010

Hershenhoren, I., Taaseh, N., Antunes, F. M., and Nelken, I. (2014). Intracellular correlates of stimulus-specific adaptation. J. Neurosci. 34, 3303-3319. doi: 10.1523/JNEUROSCI.2166-13.2014

Higgins, N. C., Storace, D. A., Escabi, M. A., and Read, H. L. (2010). Specialization of binaural responses in ventral auditory cortices. J. Neurosci. 30, 14522-14532. doi: 10.1523/JNEUROSCI.2561-10.2010

Horikawa, J., Ito, S., Hosokawa, Y., Homa, T., and Murata, K. (1988). Tonotopic representation in the rat auditory cortex. Proc. Jpn. Acad. Ser. 64, 260-263.

Horváth, J., Czigler, I., Sussman, E., and Winkler, I. (2001). Simultaneously active pre-attentive representations of local and global rules for sound sequences in the human brain. Cogn. Brain. Res. 12, 131-144. doi: 10.1016/S0926-6410(01)00038-6

Hulse, S. H., Humpal, J., and Cynx, J. (1984). Discrimination and generalization of rhythmic and arrhythmic sound patterns by european starlings music. Perception 1, 23.

Imas, O. A., Ropella, K. M., Ward, B. D., Wood, J. D., and Hudetz, A. G. (2005). Volatile anesthetics disrupt frontal-posterior recurrent information transfer at gamma frequencies in rat. Neurosci. Lett. 387, 145-150. doi: 10.1016/j.neulet.2005.06.018

Jaunmahomed, Z., and Chait, M. (2012). The timing of change detection and change perception in complex acoustic scenes. Front. Psychol. 3:396. doi: 10.3389/fpsyg.2012.00396

Kalatsky, V. A., Polley, D. B., Merzenich, M. M., Schreiner, C. E., and Stryker, M. P. (2005). Fine functional organization of auditory cortex revealed by Fourier optical imaging. Proc. Natl. Acad. Sci. U.S.A. 102, 13325-13330. doi: $10.1073 /$ pnas.0505592102

Kayser, C. (2009). Phase resetting as a mechanism for supramodal attentional control. Neuron 64, 300-302. doi: 10.1016/j.neuron.2009.10.022 
Kopell, N., Ermentrout, G. B., Whittington, M. A., and Traub, R. D. (2000). Gamma rhythms and beta rhythms have different synchronization properties. Proc. Natl. Acad. Sci. U.S.A. 97, 1867-1872. doi: 10.1073/pnas.97.4.1867

Kruglikov, S. Y., and Schiff, S. J. (2003). Interplay of electroencephalogram phase and auditory-evoked neural activity. J. Neurosci. 23, 10122-10127.

Lakatos, P., Chen, C. M., O'Connell, M. N., Mills, A., and Schroeder, C. E. (2007). Neuronal oscillations and multisensory interaction in primary auditory cortex. Neuron 53, 279-292. doi: 10.1016/j.neuron.2006.12.011

Lakatos, P., O'Connell, M. N., Barczak, A., Mills, A., Javitt, D. C., and Schroeder, C. E. (2009). The leading sense: supramodal control of neurophysiological context by attention. Neuron 64, 419-430. doi: 10.1016/j.neuron.2009.10.014

Lee, J. H., Whittington, M. A., and Kopell, N. J. (2013). Top-down beta rhythms support selective attention via interlaminar interaction: a model. PLoS Comput. Biol. 9:e1003164. doi: 10.1371/journal.pcbi.1003164

Llinás, R., Urbano, F. J., Leznik, E., Ramirez, R. R., and van Marle, H. J. (2005). Rhythmic and dysrhythmic thalamocortical dynamics: GABA systems and the edge effect. Trends Neurosci. 28, 325-333. doi: 10.1016/j.tins.2005.04.006

Makeig, S., Westerfield, M., Jung, T. P., Enghoff, S., Townsend, J., Courchesne, E., et al. (2002). Dynamic brain sources of visual evoked responses. Science 295, 690-694. doi: 10.1126/science. 1066168

Malmierca, M. S., Anderson, L. A., and Antunes, F. M. (2015). The cortical modulation of stimulus-specific adaptation in the auditory midbrain and thalamus: a potential neuronal correlate for predictive coding. Front. Syst. Neurosci. 9:19. doi: 10.3389/fnsys.2015.00019

Marella, S., and Ermentrout, G. B. (2008). Class-II neurons display a higher degree of stochastic synchronization than class-I neurons. Phys. Rev. E. 77:041918. doi: 10.1103/physreve.77.041918

Markov, N. T., Ercsey-Ravasz, M., Van Essen, D. C., Knoblauch, K., Toroczkai, Z., and Kennedy, H. (2013). Cortical high-density counterstream architectures. Science 342:1238406. doi: 10.1126/science. 1238406

Mondragón, E., Murphy, R. A., and Murphy, V. A. (2009). Rats do learn XYX rules. Anim. Behav. 78, e3-e4. doi: 10.1016/j.anbehav.2009.07.013

Murphy, M. S., and Cook, R. G. (2008). Absolute and relational control of a sequential auditory discrimination by pigeons (Columba livia). Behav. Process. 77, 210-222. doi: 10.1016/j.beproc.2007.10.008

Mustovic, H., Scheffler, K., Di Salle, F., Esposito, F., Neuhoff, J. G., Hennig, J., et al. (2003). Temporal integration of sequential auditory events: silent period in sound pattern activates human planum temporale. NeuroImage 20, 429-434. doi: $10.1016 /$ S1053-8119(03)00293-3

Nelken, I. (2014). Stimulus-specific adaptation and deviance detection in the auditory system: experiments and models. Biol. Cybern. 108, 655-663. doi: $10.1007 / \mathrm{s} 00422-014-0585-7$

Noda, T., Kanzaki, R., and Takahashi, H. (2013a). Amplitude and phaselocking adaptation of neural oscillation in the rat auditory cortex in response to tone sequence. Neurosci. Res. 79, 52-60. doi: 10.1016/j.neures.2013. 11.002

Noda, T., Kanzaki, R., and Takahashi, H. (2013b). Stimulus phase locking of cortical oscillation for auditory stream segregation in rats. PLOS ONE 8:e83544. doi: 10.1371/journal.pone.0083544

Noda, T., and Takahashi, H. (2015). Anesthetic effects of isoflurane on the tonotopic map and neuronal population activity in the rat auditory cortex. Eur. J. Neurosci. 42, 2298-2311. doi: 10.1111/ejn.13007

Ossmy, O., Fried, I., and Mukamel, R. (2015). Decoding speech perception from single cell activity in humans. Neuroimage 117, 151-159. doi: 10.1016/j.neuroimage.2015.05.001

Paradiso, G., Cunic, D., Saint-Cyr, J. A., Hoque, T., Lozano, A. M., Lang, A. E., et al. (2004). Involvement of human thalamus in the preparation of self-paced movement. Brain 127, 2717-2731. doi: 10.1093/brain/awh288

Peretz, I., Brattico, E., Järvenpää, M., and Tervaniemi, M. (2009). The amusic brain: in tune, out of key, and unaware. Brain J. Neurol. 132, 1277-1286. doi: 10.1093/brain/awp055

Perry, C., and Felsen, G. (2012). Ratss can make relative perceptual judgments about sequential stimuli. Anim. Cogn. 15, 473-481. doi: 10.1007/s10071-012-0471-4

Polley, D. B., Read, H. L., Storace, D. A., and Merzenich, M. M. (2007). Multiparametric auditory receptive field organization across five cortical fields in the albino rat. J. Neurophysiol. 97, 3621-3638. doi: 10.1152/jn.01298.2006
Ravignani, A., Sonnweber, R. S., Stobbe, N., and Fitch, W. T. (2013). Action at a distance: dependency sensitivity in a New World primate. Biol. Lett. 9:20130852. doi: 10.1098/rsbl.2013.0852

Raz, A., Grady, S. M., Krause, B. M., Uhlrich, D. J., Manning, K. A., and Banks, M. I. (2014). Preferential effect of isoflurane on top-down vs. bottom-up pathways in sensory cortex. Front. Syst. Neurosci. 8:191. doi: 10.3389/fnsys.2014.00191

Rutkowski, R. G., Miasnikov, A. A., and Weinberger, N. M. (2003). Characterisation of multiple physiological fields within the anatomical core of rat auditory cortex. Hear. Res. 181, 116-130. doi: 10.1016/S0378-5955(03)00182-5

Samaha, J., Bauer, P., Cimaroli, S., and Postle, B. R. (2015). Top-down control of the phase of alpha-band oscillations as a mechanism for temporal prediction. Proc. Natl. Acad. Sci. U.S.A. 112, 8439-8444. doi: 10.1073/pnas.1503686112

Sauseng, P., Klimesch, W., Gruber, W. R., Hanslmayr, S., Freunberger, R., and Doppelmayr, M. (2007). Are event-related potential components generated by phase resetting of brain oscillations? A critical discussion. Neuroscience 146, 1435-1444. doi: 10.1016/j.neuroscience.2007.03.014

Schneider, D. M., and Woolley, S. M. N. (2013). Sparse and backgroundinvariant coding of vocalizations in auditory scenes. Neuron 79, 141-152. doi: 10.1016/j.neuron.2013.04.038

Schroeder, C. E., Lakatos, P., Kajikawa, Y., Partan, S., and Puce, A. (2008). Neuronal oscillations and visual amplification of speech. Trends Cogn. Sci. 12, 106-113. doi: 10.1016/j.tics.2008.01.002

Schroeder, C. E., Wilson, D. A., Radman, T., Scharfman, H., and Lakatos, P. (2010). Dynamics of active sensing and perceptual selection. Curr. Opin. Neurobiol. 20, 172-176. doi: 10.1016/j.conb.2010.02.010

Shinn-Cunningham, B. G., Best, V., Dent, M. L., Gallun, F. J., McClaine, E. M., Narayan, R., et al. (2006). "Behavioral and neural identification of birdsong under several masking conditions," in Hearing - from Sensory Processing to Perception, eds B. Kollmeier, G. Klump, V. Hohmann, U. Langemann, M. Mauermann, S. Uppenkamp, and J. Verhey (Berlin; Heidelberg: SpringerVerlag), 207-214.

Shiramatsu, T. I., Kanzaki, R., and Takahashi, H. (2013). Cortical mapping of mismatch negativity with deviance detection property in rat. PLoS ONE 8:e82663. doi: 10.1371/journal.pone.0082663

Siegel, M., Donner, T. H., and Engel, A. K. (2012). Spectral fingerprints of large-scale neuronal interactions. Nat. Rev. Neurosci. 13, 121-134. doi: $10.1038 / \mathrm{nrn} 3137$

Smith, J. L., Smith, E. A., Provost, A. L., and Heathcote, A. (2010). Sequence effects support the conflict theory of $\mathrm{N} 2$ and $\mathrm{P} 3$ in the Go/NoGo task. Int. J. Psychophysiol. 75, 217-226. doi: 10.1016/j.ijpsycho.2009.11.002

Storace, D. A., Higgins, N. C., and Read, H. L. (2011). Thalamocortical pathway specialization for sound frequency resolution. J. Comp. Neurol. 519, 177-193. doi: $10.1002 / \mathrm{cne} .22501$

Taaseh, N., Yaron, A., and Nelken, I. (2011). Stimulus-specific adaptation and deviance detection in the rat auditory cortex. PLOS ONE 6:e23369. doi: 10.1371/journal.pone.0023369

Takahashi, H., Nakao, M., and Kaga, K. (2004). Cortical mapping of auditory-evoked offset responses in rats. Neuroreport 15, 1565-1569. doi: 10.1097/01.wnr.0000134848.63755.5c

Takahashi, H., Nakao, M., and Kaga, K. (2005). Interfield differences in intensity and frequency representation of evoked potentials in rat auditory cortex. Hear. Res. 210, 9-23. doi: 10.1016/j.heares.2005.05.014

Takahashi, H., Yokota, R., Funamizu, A., Kose, H., and Kanzaki, R. (2011). Learning-stage-dependent, field-specific, map plasticity in the rat auditory cortex during appetitive operant conditioning. Neuroscience 199, 243-258. doi: 10.1016/j.neuroscience.2011.09.046

Tallon-Baudry, C., and Bertrand, O. (2007). Oscillatory gamma activity in humans and its role in object representation. Trends Cogn. Sci. 3, 151-162. doi: 10.1016/S1364-6613(99)01299-1

Tallon-Baudry, C., Mandon, S., Freiwald, W. A., and Kreiter, A. K. (2004). Oscillatory synchrony in the monkey temporal lobe correlates with performance in a visual short-term memory task. Cereb. Cortex 14, 713-720. doi: 10.1093/cercor/bhh031

Toro, J. M., and Trobalón, J. B. (2005). Statistical computations over a speech stream in a rodent. Percept. Psychophys. 67, 867-875. doi: 10.3758/BF031 93539 
Traub, R. D., Whittington, M. A., Stanford, I. M., and Jefferys, J. G. R. (1996). A mechanism for generation of long-range synchronous fast oscillations in the cortex. Nature 383, 621-624. doi: 10.1038/383621a0

Urcelay, G. P., and Miller, R. R. (2010). On the generality and limits of abstraction in rats and humans. Anim. Cogn. 13, 21-32. doi: 10.1007/s10071-009-0295-Z

Vanvooren, S., Hofmann, M., Poelmans, H., Ghesquiere, P., and Wouters, J. (2015). Theta, beta and gamma rate modulations in the developing auditory system. Hear. Res. 327, 153-162. doi: 10.1016/j.heares.2015.06.011

von Stein, A., and Sarnthein, J. (2000). Different frequencies for different scales of cortical integration: from local gamma to long range alpha/theta synchronization. Int. J. Psychophysiol. 38, 301-313. doi: 10.1016/S0167-8760(00)00172-0

Wacongne, C., Changeux, J. P., and Dehaene, S. (2012). A neuronal model of predictive coding accounting for the mismatch negativity. J. Neurosci. 32, 3665-3678. doi: 10.1523/JNEUROSCI.5003-11.2012

Wacongne, C., Labyt, E., van Wassenhove, V., Bekinschtein, T., Naccache, L., and Dehaene, S. (2011). Evidence for a hierarchy of predictions and prediction errors in human cortex. Proc. Natl. Acad. Sci. U.S.A. 108, 20754-20759. doi: $10.1073 /$ pnas. 1117807108

Wang, J., and Salvi, R. (2002). Gamma-aminobutyric acid circuits shape response properties of auditory cortex neurons. Brain Res. 944, 219-231. doi: 10.1016/S0006-8993(02)02926-8

Wang, X. J. (2010). Neurophysiological and computational principles of cortical rhythms in cognition. Physiol. Rev. 90, 1195-1268. doi: 10.1152/physrev.00035.2008

Watrous, A. J., Fell, J., Ekstrom, A. D., and Axmacher, N. (2015). More than spikes: common oscillatory mechanisms for content specific neural representations during perception and memory. Curr. Opin. Neurobiol. 31, 33-39. doi: 10.1016/j.conb.2014.07.024

Wehr, M., and Zador, A. M. (2005). Synaptic mechanisms of forward suppression in rat auditory cortex. Neuron 47, 437-445. doi: 10.1016/j.neuron.2005.06.009
Wilson, B., Slater, H., Kikuchi, Y., Milne, A. E., Marslen-Wilson, W D., Smith, K., et al. (2013). Auditory artificial grammar learning in macaque and marmoset monkeys. J. Neurosci. 33, 18825-18835. doi: 10.1523/JNEUROSCI.2414-13.2013

Wilson, B., Smith, K., and Petkov, C. I. (2015). Mixed-complexity artificial grammar learning in humans and macaque monkeys: evaluating learning strategies. Eur. J. Neurosci. 41, 568-578. doi: 10.1111/ejn.12834

Wimber, M., Maass, A., Staudigl, T., Richardson-Klavehn, A., and Hanslmayr, S. (2012). Rapid memory reactivation revealed by oscillatory entrainment. Curr. Biol. 22, 1482-1486. doi: 10.1016/j.cub.2012.05.054

Winkler, I., Denham, S. L., and Nelken, I. (2009). Modeling the auditory scene: predictive regularity representations and perceptual objects. Trends Cogn. Sci. 13, 532-540. doi: 10.1016/j.tics.2009.09.003

Yokota, R., Aihara, K., Kanzaki, R., and Takahashi, H. (2015). Learning-stagedependent plasticity of temporal coherence in the auditory cortex of rats. Brain Topogr. 28, 401-410. doi: 10.1007/s10548-014-0359-5

Zhang, L. I., Tan, A. Y., Schreiner, C. E., and Merzenich, M. M. (2003). Topography and synaptic shaping of direction selectivity in primary auditory cortex. Nature 424, 201-205. doi: 10.1038/nature01796

Conflict of Interest Statement: The authors declare that the research was conducted in the absence of any commercial or financial relationships that could be construed as a potential conflict of interest.

Copyright (c) 2017 Noda, Amemiya, Shiramatsu and Takahashi. This is an openaccess article distributed under the terms of the Creative Commons Attribution License (CC BY). The use, distribution or reproduction in other forums is permitted, provided the original author(s) or licensor are credited and that the original publication in this journal is cited, in accordance with accepted academic practice. No use, distribution or reproduction is permitted which does not comply with these terms. 